\title{
Credibility Beyond Replicability: Improving the Four Validities in Psychological Science
}

\author{
Simine Vazire ${ }^{1}$, Sarah R. Schiavone ${ }^{2}$, and Julia G. Bottesini ${ }^{2}$ \\ ${ }^{1}$ Melbourne School of Psychological Sciences, University of Melbourne, Australia \\ ${ }^{2}$ University of California, Davis, USA
}

\begin{abstract}
Psychological science's "credibility revolution" has produced an explosion of metascientific work on improving research practices. While much attention has been paid to replicability (reducing false positives), improving credibility depends on addressing a wide range of problems afflicting psychological science, beyond simply making psychology more replicable. Here we focus on the "four validities" (Shadish et al., 2002) and highlight recent developments aimed at improving these four validities in psychology research, many of which have been led by early career researchers. We propose that the credibility revolution in psychology, which has its roots in replicability, can be harnessed to help psychology improve its validity more broadly.
\end{abstract}

Keywords: metascience, credibility revolution, replication crisis, four validities

Psychology's 'replication crisis' produced a crisis of credibility that propelled many efforts to improve the quality of psychological science, sometimes called the 'credibility revolution' (Vazire, 2018). It is difficult to define the boundaries of this movement, but it is characterized by collaborative, grassroots efforts, often driven by early career researchers (Maizey \& Tzavella, 2019). This movement has been facilitated by new organizations; those with the most noticeable impact in psychology include the Center for Open Science (established 2013), the Society for the Improvement of Psychological Science (established 2016), the Psychological Science Accelerator (established 2017), and the ReproducibiliTea network (established 2018).

Much of the work initially focused on what seemed to be the most pressing problem: large-scale replication attempts only rarely produced results consistent with the original studies. These difficulties replicating the results of pub-

Conflict of Interest statement: All authors identify metascience as one of their primary fields and are involved in the reform movement described in this paper. Simine Vazire was a co-founder and past president of the Society for the Improvement of Psychological Science.

Acknowledgements: This work was supported by a NSF Graduate Research Fellowship (\#1247392) to Sarah R. Schiavone.

Author contributions: Authors contributed in descending order of authorship. All authors approve the final version of this manuscript.

Corresponding author: Simine Vazire; simine@gmail.com lished-and often highly influential - studies pointed to a potential problem, namely, many of our published findings may be false discoveries. Reducing the prevalence of false positive findings, and increasing the replicability of our literature, became a prominent goal. However, credibility depends on the quality of research practices more broadly, not just the replicability of research findings. Thus, the scope of the credibility revolution is much broader than simply addressing replicability concerns.

The early focus on replicability was a reasonable response given the shock to the system delivered by the many highprofile replication failures. Replicability is a very low bar, and passing this bar alone means very little-many confounded effects are replicable, as are grossly misinterpreted effects, artifacts, and other errors. Failing to pass this bar, however, usually means that there is little more to discuss. Thus, acceptable levels of replicability are a prerequisite to credibility, but replicability is not sufficient for credibility. Accordingly, improving the credibility of psychological science requires attention to all aspects of validity.

In line with this, many advances from the credibility revolution have targeted research quality more broadly, not just replicability. For example, Registered Reports (in which formal peer review happens and an editorial decision is made before data are collected) provide an opportunity to receive feedback on the conceptualization, design, and execution of the research when that feedback can still be incorporated into the research plan, potentially improving many different aspects of research quality.

Here, we highlight recent work that draws attention to aspects of research quality beyond replicability (for a review of 
recent work on replicability and reproducibility, see Nosek et al., in press), organized around the "four validities": construct validity, internal validity, external validity, and statistical conclusion validity (Shadish et al., 2002). We highlight some of the most important work from the last ten years aimed at drawing attention to threats to each of the four validities, and developing solutions. Some of these contributions are new developments, whereas others are attempts to bring new life to old ideas that warrant greater attention and uptake. Because this is a grassroots movement, it is impossible to say which developments are or are not part of the movement (or were or were not made possible by the zeitgeist fostered by the movement). Given these fuzzy boundaries, we highlight projects that were developed 1) since 2011,2 ) primarily by psychologists, and 3) often by early career researchers.

We aim to capture the leading edge of the reform movement in psychology. As such, there is much we do not cover here, including the classics (see also Kenny, 2019). We also do not cover the literature on systemic threats to validity, such as incentives and peer review. Finally, we skip early work in the reform movement on questionable research practices and transparency (for an overview, see Nelson et al., 2018). Instead, we focus on the "next generation" developments that build on these foundations to improve the construct, internal, external, and statistical conclusion validity of psychology research.

\section{Construct Validity}

Construct validity refers to the validity of inferences made about how the measured or manipulated variables relate to the constructs of interest. Social scientists frequently skip important steps in the definition and conceptualization of the constructs we study (Alexandrova \& Haybron, 2016). This lack of conceptual clarity, or mismatch between operationalizations and constructs, threatens the validity of our inferences.

These threats were vividly demonstrated in a project in which fifteen independent research teams designed studies to test five research questions provided in conceptual form (i.e., without specific operationalizations; Landy et al., 2020). The teams' results and conclusions varied dramatically for four of the five research questions. For example, for the research question "When directly asked, do people explicitly self-report an awareness of harboring negative automatic associations with members of negatively stereotyped social groups?", about half of the research teams found strong evidence that people do self-report harboring such associations, and half found evidence that people deny harboring such associations. This is not surprising when looking at the diversity of teams' operationalizations of the variables "negative automatic associations" and "negatively stereotyped groups" (see pp. 11-36 in https://osf.io/fn7za/). Without conceptual clarity about the meaning of the constructs, there is no way to judge which specific designs and operationalizations are producing valid results.

The challenges do not end with conceptual clarity-designing a valid measure or manipulation is notoriously challenging (Flake \& Fried, 2020). In a survey of published research in social and personality psychology, only about half of scales used were accompanied by a citation (Flake et al., 2017), suggesting many were developed ad hoc, or were not appropriately cited. Moreover, multi-item scales were often reported with no information about validity. Similar results have been found regarding the reported psychometric properties of 15 of the most commonly used measures in social and personality psychology (Hussey \& Hughes, 2020). These results suggest that measurement validity is quite weak in much of psychology research. This can not only attenuate effect sizes, but lead to spurious effects and inflated estimates.

Even when measures are 'validated' following common recommendations for construct validation, it is unclear whether they are actually valid. In a striking demonstration of this, Satchel and colleagues (2020) created a measure of "offline-friend addiction" by modifying a popular measure of problematic social media usage. For example, the items "I have spent more time with friends than I initially intended", "I spend time with friends to forget about personal problems", and "I often think about my friends when I'm not with them" were adapted from nearly identical items about Facebook use on Facebook addiction scales. The authors then 'validated' their offline friend addiction scale using conventional construct validation techniques, such as reporting the factor structure, test-retest reliability, and convergent validity of the measure. Then, using cutoffs typical in the addiction literature, they report that their measure would classify $69 \%$ of study participants as being "addicted to spending time with their friends".

The authors use this demonstration to make the case that the typical standards for validating addiction measures are inadequate. The issues range from insufficient attention to conceptual issues (e.g., is repetitive behavior always problematic?) to the inadequacy of statistical practices used to establish validity (e.g., what looks like convergent validity could actually be driven by response styles). While the authors limit their conclusions to problematic norms in the subfields of addiction and social media research, we believe these norms are fairly widespread.

Concerns about the lack of validation and inadequacy of validation techniques can also be raised regarding the validity of experimental manipulations, and this has also gotten renewed attention, including a vigorous debate about 
the uses and misuses of manipulation checks (e.g., Ejelöv \& Luke, 2020; Gruijters, 2020). Similarly, behavioral, cognitive, and physiological measures are often assumed to be valid without proper verification. For example, researchers often use behavioral assessments of cognitive processes (e.g., dot-probe tasks) without reporting their reliability, let alone evidence of their validity (Parsons, Kruijt, \& Fox, 2019).

The themes central to construct validity-defining constructs and establishing the validity of measures and manipulations-are also prominently featured in some broader developments in the reform movement. For example, Registered Reports emphasize that proposals should be evaluated in part on the rigor of the design, including the construct validity of the measured and manipulated variables (Chambers \& Tzavella, 2020). Similarly, the Psychological Science Accelerator emphasizes the importance of construct validity in the evaluation of study proposals. Moreover, their unique collaborative network enables them to explore new frontiers in construct validity, such as assessing cross-site measurement invariance (Moshontz et al., 2018).

\section{Internal Validity}

Internal validity is the validity of causal inferences: Are assumptions upon which causal inferences are based explicitly stated and justified? Have plausible alternative explanations been convincingly ruled out? Psychology lags behind many other social and behavioral sciences in this domain.

Psychology researchers often assume that experimental designs ensure internal validity. However, even in a controlled experiment, there are many potential threats to internal validity. Some of these threats have received renewed attention in recent years. For example, the problem of selective attrition was recently demonstrated to be a serious threat, particularly in online studies. Zhou and Fischbach (2016) demonstrated this threat vividly by conducting online studies using methods typical in social psychology. First, they found that attrition is very high, ranging from $30 \%$ to $50 \%$ in six common experimental paradigms. More importantly, they show that attrition is not equal across experimental conditions-different numbers and types of people persist in different conditions. Next, they conduct original research to show that selective attrition can produce exciting but spurious results. For example, they show that selective attrition produces a result that appears to suggest that support for gun restrictions can be increased simply by asking people to write an essay explaining the reasons for their views on gun restrictions. This happens because people's preexisting views on gun restrictions predict attrition differently in the two conditions (writing vs. control) such that, by the time the DV (post-manipulation support for gun restrictions) is measured, there are a higher proportion of people who support gun restrictions in the writing condition than the control condition, introducing a confound. The authors point out that this artifact could easily be mistaken for a real effect. This could wreak havoc on theory development and in real-world applications such as political strategy. This threat is vastly overlooked in some areas of psychology research, judging by the little attention paid to attrition in published studies. For example, Zhou and Fishback found that of the 289 published reports of online social and personality psychology studies that they examined, only $2 \%$ reported levels of attrition. $\mathrm{Fu}-$ ture metascientific studies will reveal whether this situation has improved in recent years.

Other recent papers have similarly raised awareness about threats to causal inference that show up even in the context of true experiments. For example, Wiernik and Dahlke (2020) highlight how effect sizes in experimental studies can by influenced by the response variability in that particular sample, leading to heterogeneous results across even very similar studies. Fiedler and colleagues (2018), provided a powerful reminder that even in the context of a true experiment, mediation analyses cannot establish the correct causal model-there will always need to be explicit theorizing about alternate causal models (e.g., alternate mediators). Their review of recent psychology papers found that $14 \%$ present an a priori theoretical argument for the proposed mediation model, and only $9 \%$ test alternative causal models. While these issues have been known for years, many recent papers provide concrete demonstrations of the seriousness of the threats, empirical data on the prevalence of the problem, and offer solutions.

These and other threats to internal validity are also present in observational (i.e., non-experimental) studies. These problems may seem less important because authors of papers reporting on observational studies often state that a limitation of their design is that it prevents them from drawing causal inferences. If no causal inferences are made or implied, then internal validity is irrelevant. However, our impression is that this restraint is often shown only in the limitations section-the same reports seem to often use causal language throughout the rest of the paper.

Responsible causal inferences are possible with observational studies, but they are challenging and rare. Achieving internal validity requires being upfront about the fact that we are interested in causal mechanisms, and about the obstacles that stand in the way. Here, too, attention to this issue seems to be growing: A recent paper on "thinking clearly about correlations and causation", which is already widely cited and discussed, provides an overview of the challenges and possible solutions to causal inference in observational studies (Rohrer, 2018). This shift, from simply apologizing 
for the non-experimental design in the limitations section while continuing to make causal claims elsewhere in the paper, to thinking more about when causal inferences are or are not warranted, is long overdue. We need to move towards a more thoughtful treatment of internal validity in both experimental and non-experimental contexts.

\section{External Validity}

External validity is the validity of inferences about how the observed effect will generalize beyond the specific conditions of the study. This includes generalizations to other people (or populations of whatever unit was sampled, if not people), other stimuli, other operationalizations or research designs, and other settings or times.

The poor state of psychological research with respect to unwarranted generalizations made from convenience samples of mostly North American undergraduate students has received a lot of attention. However, there has been little evidence of progress on this front (Pollet \& Saxton, 2019). Meanwhile, evidence that WEIRD samples are a serious threat to external validity continues to accumulate. For example, Tiokhin and colleagues (2019) tested an effect that had been well-replicated in WEIRD samples, social discounting, in low-literacy and resource-scarce settings in Bangladesh and India. According to social discounting, people should be more generous towards socially close than socially distant others. This was not the case in these two novel settings. This lack of generalizability provides rich fodder for theorizing about human behavior.

The rapid growth of crowdsourced research in psychology has finally made a dent in the problem of overreliance on WEIRD samples. Examples include the Many Labs projects (Klein et al., 2018) and the Psychological Science Accelerator (PSA; Moshontz et al., 2018), with over 1,194 collaborators in 80 countries. Crowdsourced research allows not just for more generalizable claims, but also the systematic examination of heterogeneity and context-specificity of findings across countries, languages, and cultures. Moreover, it provides an opportunity to test generalizability across populations of stimuli, settings, or procedures. Given these many benefits of team science, we hope the PSA serves as an inspiration for similar team science efforts, and that the scientific community invests in these efforts.

Generalizability across seemingly-trivial variations in the research design (e.g., stimuli, experimenters, procedures, etc.) is an often overlooked aspect of external validity. However, it has attracted renewed attention in recent years, most notably in Yarkoni's (2019) paper drawing attention to the many difficulties in making generalizable claims. One solution to this problem is to systematically vary levels of these factors and model them as random effects. Others emphasize the need to select stimuli and levels of variables that are representative of those found in the real world. Calls to pay more attention to this issue go back at least to the 1960s, but have had little effect beyond psycholinguistics. Perhaps the renewed attention sparked by Yarkoni's paper, combined with the opportunities presented by efforts like the PSA and Many Labs, will lead to sustained change this time around.

\section{Statistical Conclusion Validity}

Of the four validities reviewed here, statistical conclusion validity-or the validity of statistical inferences-has received the most attention from the reform movement (e.g., tools such as GRIM (Brown \& Heathers, 2017) and statcheck (Epskamp \& Nuijten, 2018); discussions around p-hacking, HARKing, data sharing, and reproducibility). It is impossible to do justice to this literature in this short review. Here, we focus on developments that question and clarify the foundations of statistical inference in psychological research, and aim to bolster our ability to make valid, intersubjectively verifiable statistical inferences.

There are many assumptions necessary to make the leap from statistical result to inference, and these are often overlooked. The consequences of this leap were starkly illustrated in two projects in which analysts were given datasets and asked to draw statistical inferences from them. In both cases (Silberzahn et al., 2018; Starns et al., 2019), very different-in some cases contradictory-statistical conclusions and inferences were drawn from the same datasets.

While many of these differences are attributable to surfacelevel statistical practices, the problem may run much deeper. Scheel and colleagues (2020) recently pointed out that, even though much research in psychology takes the form of hypothesis testing, we often lack the groundwork necessary to perform high quality hypothesis tests (e.g., clear concepts, valid measures, articulated auxiliary assumptions, precise statistical predictions). To strengthen the basis for drawing conceptual inferences from statistical results, Tunç and Tunç (2019) have proposed a framework that provides a principled way to disentangle the possible interpretations of statistical results (e.g., when to attribute a negative result to the central vs. auxiliary hypotheses) while preserving severe testing of the theoretical hypothesis.

Other projects focus on addressing more specific challenges, typically within the framework of hypothesis testing, such as establishing evidence for absence of an effect (Fidler et al., 2018) or determining a "smallest effect size of interest" (Anvari \& Lakens, 2019). Finally, Registered Reports represent a major advance for statistical conclusion validity. The aim of Registered Reports is to reduce bias by eliminating 
many of the avenues for undisclosed flexibility in research. In a recent study examining Registered Reports, only $44 \%$ presented a positive key result, compared to $96 \%$ in the traditional literature (Scheel et al., 2021). There are, of course, many potential explanations for this discrepancy, but one likely hypothesis is that the traditional literature is heavily biased by questionable research and publication practices. The checks imposed on authors and journals by Registered Reports help reduce these biases.

\section{Conclusion}

The credibility revolution in psychology was initially fueled largely by concerns about false positives and a desire to boost the replicability of psychology findings. Many of the efforts stemming from the credibility revolution (e.g., Registered Reports, expanding team science) also have the potential to address threats to other aspects of validity, including fundamental issues related to construct validity, internal validity, external validity, and statistical inference. The last ten years have seen heightened levels of activity and interest in research methods and practices among the community of psychology researchers. Much of this has been led by early career researchers working collaboratively outside of pre-existing institutions. The issues that have been raised and solutions that have been developed have the potential to substantially improve how psychology research is practiced, and how it is published and communicated.

Developments such as those highlighted above, combined with renewed attention to existing resources such as graphical causal models, metascience, and replication, have paved the way for improvements beyond just the replicability of our findings. This is in line with the animating drive behind the credibility revolution: to bolster our credibility as a scientific field through rigorous, high quality research.

\section{References}

Alexandrova, A., \& Haybron, D. M. (2016). Is Construct Validation Valid? Philosophy of Science, 83(5), 1098-1109. https://doi.org/10.1086/687941

Anvari, F., \& Lakens, D. (2019). Using Anchor-Based Methods to Determine the Smallest Effect Size of Interest (Preprint). PsyArXiv. https://doi.org/10.31234/osf. io/syp5a

Brown, N. J., \& Heathers, J. A. (2017). The grim test: A simple technique detects numerous anomalies in the reporting of results in psychology. Social Psychological and Personality Science, 8(4), 363-369.

Chambers, C. D., \& Tzavella, L. (2020). Registered Reports: Past, Present and Future (Preprint). MetaArXiv. https: //doi.org/10.31222/osf.io/43298
Ejelöv, E., \& Luke, T. J. (2020). "Rarely safe to assume": Evaluating the use and interpretation of manipulation checks in experimental social psychology. Journal of Experimental Social Psychology, 87, 103937. https: //doi.org/10.1016/j.jesp.2019.103937

Epskamp, S., \& Nuijten, M. (2014). Statcheck: Extract statistics from articles and recompute $\mathrm{p}$ values $(\mathrm{r}$ package version 1.0. 0.)

Fidler, F., Singleton Thorn, F., Barnett, A., Kambouris, S., \& Kruger, A. (2018a). The Epistemic Importance of Establishing the Absence of an Effect. Advances in Methods and Practices in Psychological Science, 1(2), 237-244. https://doi.org/10.1177/2515245918770407

Fidler, F., Singleton Thorn, F., Barnett, A., Kambouris, S., \& Kruger, A. (2018b). The epistemic importance of establishing the absence of an effect. Advances in Methods and Practices in Psychological Science, 1(2), 237-244.

Fiedler, K., Harris, C., \& Schott, M. (2018). Unwarranted inferences from statistical mediation tests - An analysis of articles published in 2015. Fournal of Experimental Social Psychology, 75, 95-102. https://doi.org/10. 1016/j.jesp.2017.11.008

Flake, J. K., Pek, J., \& Hehman, E. (2017). Construct Validation in Social and Personality Research: Current Practice and Recommendations. Social Psychological and Personality Science, 8(4), 370-378. https : //doi.org/10.1177/1948550617693063

Flake, J. K., \& Fried, E. I. (2019). Measurement Schmeasurement: Questionable Measurement Practices and How to Avoid Them (Preprint). PsyArXiv. https://doi.org/ 10.31234/osf.io/hs7wm

Gruijters, S. L. (2020). The Fallacy of Manipulation "Checks" in Psychological Experiments (Preprint). PsyArXiv. https://doi.org/10.31234/osf.io/fkzv5

Hussey, I., \& Hughes, S. (2020). Hidden Invalidity Among 15 Commonly Used Measures in Social and Personality Psychology. Advances in Methods and Practices in Psychological Science, 3(2), 166-184. https://doi.org/ $10.1177 / 2515245919882903$

Kenny, D. A. (2019). Enhancing validity in psychological research. American Psychologist, 74(9), 1018-1028. https://doi.org/10.1037/amp0000531

Klein, R. A., Vianello, M., Hasselman, F., Adams, B. G., Adams, R. B., Alper, S., Aveyard, M., Axt, J. R., Babalola, M. T., Bahník, Š., Batra, R., Berkics, M., Bernstein, M. J., Berry, D. R., Bialobrzeska, O., Binan, E. D., Bocian, K., Brandt, M. J., Busching, R., ... Nosek, B. A. (2018). Many Labs 2: Investigating Variation in Replicability Across Samples and Settings. Advances in Methods 
and Practices in Psychological Science, 1(4), 443-490. https://doi.org/10.1177/2515245918810225

Landy, J. F., Jia, M. (, Ding, I. L., Viganola, D., Tierney, W., Dreber, A., Johannesson, M., Pfeiffer, T., Ebersole, C. R., Gronau, Q. F., Ly, A., van den Bergh, D., Marsman, M., Derks, K., Wagenmakers, E.-J., Proctor, A., Bartels, D. M., Bauman, C. W., Brady, W. J., ... Uhlmann, E. L. (2020). Crowdsourcing hypothesis tests: Making transparent how design choices shape research results. Psychological Bulletin, 146(5), 451479. https://doi.org/10.1037/bul0000220

Maizey, L., \& Tzavella, L. (2019). Barriers and solutions for early career researchers in tackling the reproducibility crisis in cognitive neuroscience. Cortex, 113, 357359. https://doi.org/10.1016/j.cortex.2018.12.015

Moshontz, H., Campbell, L., Ebersole, C. R., IJzerman, H., Urry, H. L., Forscher, P. S., Grahe, J. E., McCarthy, R. J., Musser, E. D., Antfolk, J., Castille, C. M., Evans, T. R., Fiedler, S., Flake, J. K., Forero, D. A., Janssen, S. M. J., Keene, J. R., Protzko, J., Aczel, B., ... Chartier, C. R. (2018). The Psychological Science Accelerator: Advancing Psychology Through a Distributed Collaborative Network. Advances in Methods and Practices in Psychological Science, 1(4), 501-515. https: //doi.org/10.1177/2515245918797607

Nelson, L. D., Simmons, J., \& Simonsohn, U. (2018). Psychology's Renaissance. Annual Review of Psychology, 69(1), 511-534. https://doi.org/10.1146/annurevpsych-122216-011836

Nosek, B. A., Hardwicke, T. E., Moshontz, H., Allard, A., Corker, K. S., Almenberg, A. D., Fidler, F., Hilgard, J., Kline, M., Nuijten, M. B., et al. (2021). Replicability, robustness, and reproducibility in psychological science.

Parsons, S., Kruijt, A.-W., \& Fox, E. (2019). Psychological science needs a standard practice of reporting the reliability of cognitive-behavioral measurements. Advances in Methods and Practices in Psychological Science, 2(4), 378-395.

Pollet, T. V., \& Saxton, T. K. (2019). How Diverse Are the Samples Used in the Journals 'Evolution \& Human Behavior' and 'Evolutionary Psychology'? Evolutionary Psychological Science, 5(3), 357-368. https: //doi.org/10.1007/s40806-019-00192-2

Rohrer, J. M. (2018). Thinking Clearly About Correlations and Causation: Graphical Causal Models for Observational Data. Advances in Methods and Practices in Psychological Science, 1(1), 27-42. https://doi.org/10. $1177 / 2515245917745629$

Satchell, L. P., Fido, D., Harper, C. A., Shaw, H., Davidson, B., Ellis, D. A., Hart, C. M., Jalil, R., Bartoli, A. J.,
Kaye, L. K., Lancaster, G. L. J., \& Pavetich, M. (2020). Development of an Offline-Friend Addiction Questionnaire (O-FAQ): Are most people really social addicts? Behavior Research Methods. https://doi.org/ 10.3758/s13428-020-01462-9

Scheel, A. M., Schijen, M. R., \& Lakens, D. (2021). An excess of positive results: Comparing the standard psychology literature with registered reports. Advances in Methods and Practices in Psychological Science, 4(2), 25152459211007467.

Scheel, A. M., Tiokhin, L., Isager, P. M., \& Lakens, D. (2020). Why hypothesis testers should spend less time testing hypotheses. Perspectives on Psychological Science, 1745691620966795.

Shadish, W. R., Cook, T. D., \& Campbell, D. T. (2002). Experimental and Quasi-experimental Designs for Generalized Causal Inference. Houghton Mifflin.

Silberzahn, R., Uhlmann, E. L., Martin, D. P., Anselmi, P., Aust, F., Awtrey, E., Bahník, Š., Bai, F., Bannard, C., Bonnier, E., Carlsson, R., Cheung, F., Christensen, G., Clay, R., Craig, M. A., Dalla Rosa, A., Dam, L., Evans, M. H., Flores Cervantes, I., ... Nosek, B. A. (2018). Many Analysts, One Data Set: Making Transparent How Variations in Analytic Choices Affect Results. Advances in Methods and Practices in Psychological Science, 1(3), 337-356. https:// doi.org/10.1177/ 2515245917747646

Starns, J. J., Cataldo, A. M., Rotello, C. M., Annis, J., Aschenbrenner, A., Bröder, A., Cox, G., Criss, A., Curl, R. A., Dobbins, I. G., Dunn, J., Enam, T., Evans, N. J., Farrell, S., Fraundorf, S. H., Gronlund, S. D., Heathcote, A., Heck, D. W., Hicks, J. L., ... Wilson, J. (2019). Assessing Theoretical Conclusions With Blinded Inference to Investigate a Potential Inference Crisis. Advances in Methods and Practices in Psychological Science, 2(4), 335-349. https:// doi.org/10.1177/ 2515245919869583

Tiokhin, L., Hackman, J., Munira, S., Jesmin, K., \& Hruschka, D. (2019). Generalizability is not optional: Insights from a cross-cultural study of social discounting. Royal Society Open Science, 6(2), 181386. https://doi. org/10.1098/rsos.181386

Tunç, D. U., \& Tunç, M. N. (2020). A Falsificationist Treatment of Auxiliary Hypotheses in Social and Behavioral Sciences: Systematic Replications Framework (Preprint). PsyArXiv. https://doi.org/10.31234/osf.io/pdm7y

Vazire, S. (2018). Implications of the credibility revolution for productivity, creativity, and progress. Perspectives on Psychological Science, 13(4), 411-417.

Wiernik, B. M., \& Dahlke, J. A. (2020). Obtaining Unbiased Results in Meta-Analysis: The Importance of Cor- 
recting for Statistical Artifacts. Advances in Methods and Practices in Psychological Science, 3(1), 94-123. https://doi.org/10.1177/2515245919885611

Yarkoni, T. (2019). The Generalizability Crisis (Preprint). PsyArXiv. https://doi.org/10.31234/osf.io/jqw35

Zhou, H., \& Fishbach, A. (2016). The pitfall of experimenting on the web: How unattended selective attrition leads to surprising (yet false) research conclusions. fournal of Personality and Social Psychology, 111(4), 493-504. https://doi.org/10.1037/pspa0000056

\section{Recommended Readings}

Munafò, M. R., Nosek, B. A., Bishop, D. V. M., Button, K. S., Chambers, C. D., Sert, N. P. D., Simonsohn, U., Wagenmakers, E.-J., Ware, J. J., Ioannidis, J. P. A., \& et al. (2017). A manifesto for reproducible science. Nature Human Behaviour, 1(1). https://doi.org/10. 1038/s41562-016-0021

Nelson, L. D., Simmons, J., \& Simonsohn, U. (2018). Psychology's Renaissance. Annual Review of Psychology, 69(1), 511-534. https://doi.org/10.1146/annurevpsych-122216-011836

Scheel, A. M., Schijen, M. R., \& Lakens, D. (2021). An excess of positive results: Comparing the standard psychology literature with registered reports. Advances in Methods and Practices in Psychological Science, 4(2), 25152459211007467.

Yarkoni, T. (2019). The Generalizability Crisis (Preprint). PsyArXiv. https://doi.org/10.31234/osf.io/jqw35 